Review Article

\title{
Monitoring Minimal Residual Disease in the Myeloproliferative Neoplasms: Current Applications and Emerging Approaches
}

\author{
Karl Haslam and Stephen E. Langabeer \\ Cancer Molecular Diagnostics, St. James's Hospital, Dublin 8, Ireland \\ Correspondence should be addressed to Karl Haslam; khaslam@stjames.ie
}

Received 8 September 2016; Accepted 5 October 2016

Academic Editor: Salah Aref

Copyright ( 92016 K. Haslam and S. E. Langabeer. This is an open access article distributed under the Creative Commons Attribution License, which permits unrestricted use, distribution, and reproduction in any medium, provided the original work is properly cited.

\begin{abstract}
The presence of acquired mutations within the JAK2, CALR, and MPL genes in the majority of patients with myeloproliferative neoplasms (MPN) affords the opportunity to utilise these mutations as markers of minimal residual disease (MRD). Reduction of the mutated allele burden has been reported in response to a number of therapeutic modalities including interferon, JAK inhibitors, and allogeneic stem cell transplantation; novel therapies in development will also require assessment of efficacy. Realtime quantitative PCR has been widely adopted for recurrent point mutations with assays demonstrating the specificity, sensitivity, and reproducibility required for clinical utility. More recently, approaches such as digital PCR have demonstrated comparable, if not improved, assay characteristics and are likely to play an increasing role in MRD monitoring. While next-generation sequencing is increasingly valuable as a tool for diagnosis of MPN, its role in the assessment of MRD requires further evaluation.
\end{abstract}

\section{Introduction}

The Philadelphia chromosome-negative myeloproliferative neoplasms (MPN) are clonal hematopoietic diseases characterised by bone marrow proliferation of one or more of the myeloid cell lineages with no marked alterations in cellular maturation. MPNs classically comprise the clinically and pathologically related polycythemia vera (PV), essential thrombocythemia (ET), and primary myelofibrosis (PMF). In PV and ET, the potential exists for the disease to transform to a myelofibrotic stage and, together with PMF, transform to acute myeloid leukemia. Identification of the JAK2 V617F mutation, now over a decade ago, revolutionised the molecular diagnosis of MPN as this mutation is present in up to $95 \%$ of patients with $\mathrm{PV}$ and in approximately $50-60 \%$ of patients with ET and PMF. Subsequent identification of further disease initiating mutations, such as those in JAK2 exon 12, MPL exon 10, and CALR exon 9, allows the implementation of molecular diagnostic algorithms that are able to identify a clonal marker of disease in the vast majority of classical MPN patients. Numerous other myeloid-associated mutations have been additionally detected in MPN patients whose acquisition appears to influence the phenotype and disease course [1]. Several laboratory approaches are available for the identification of these mutations at diagnosis with selection of methodology largely dependent on the clinical utility required [2].

The primary treatment goals in MPN are to avoid thrombosis and bleeding, treat MPN related symptoms, improve quality of life, and minimize risk of malignant transformation and/or post-ET/PV myelofibrosis. However, the presence of the JAK2, CALR, and MPL "driver" mutations allows them to be utilised for diagnosis, but quantitatively, they may serve as markers of minimal residual disease (MRD), an additional, valuable indicator of depth of response to therapeutic intervention. To date, the clinical validity of determining JAK2, CALR, and MPL MRD responses has been demonstrated with several modalities including interferon alpha, JAK 1/2 inhibitors, and allogeneic stem cell transplantation (ASCT). Novel targeted agents for MPN in development include specific JAK2 inhibitors, histone deacetylase inhibitors, hypomethylating agents, heat shock protein 90 inhibitors, PI3-AKT-mTOR inhibitors, and telomerase inhibitors, all of which, alone or in combination 
with established therapies, will require assessment of efficacy [3].

The presence of recurrent point mutations facilitates the use of allele-specific quantitative PCR (qPCR) which has become widely adopted. Emerging approaches and platforms that are becoming increasingly implemented in the diagnostic setting with potential application for MRD monitoring include digital PCR (dPCR) and next-generation sequencing (NGS). This review will provide a brief overview of those methods already integrated into practice and consider those in development in regard to their clinical applicability to monitor MRD with respect to the three main types of MPN driver mutations.

\section{Current Applications}

Initial Sanger sequencing was superseded by pyro-sequencing, melt curve analysis, and allele-specific PCR for routine diagnostics because of its limited sensitivity and therefore inability to detect low mutation allele burdens. Remaining diagnostic methodologies vary in their sensitivity but should allow for the routine detection of the JAK2 V617F or MPL exon 10 point mutations at an allele burden of approximately $1-3 \%[4,5]$. Assessment of MRD requires quantitation over a dynamic range of at least three logs, and taking heed of the acceptance of $B C R-A B L 1$ monitoring into the routine clinical management of chronic myeloid leukemia, a variety of allelespecific qPCR approaches have been widely adopted. Comparative studies have demonstrated the superior sensitivity of qPCR especially after calibration to common standards $[6,7]$. Several primer/probe combinations have been used to quantitate the JAK2 V617F allele burden by qPCR $[8,9]$ but assays can vary markedly in their performance. In order to address this issue and establish a consistent approach, a network of centres systematically evaluated several qPCR assays and was able to recommend the most consistently performing assay, suitable for assessing response in clinical trials, predicting outcome and guiding management of patients post-ASCT [10]. Mutations of MPL exon 10 are present in approximately $5 \%$ of ET and $<10 \%$ of PMF patients. This low frequency, compared to the JAK2 V617F and CALR exon 9 mutations, may explain that while several, sensitive qPCRbased techniques have been described for detection of the $M P L$ exon 10 mutations at diagnosis [11-13], there remains a lack of information regarding their utility as markers of MRD in these MPN. Insertion/deletion (indel) mutations of CALR exon 9 are the most recently discovered MPN driver mutations. While semiquantitative, diagnostic fragment length analysis (FLA) has been extensively embraced, qPCR methods have been hampered by the variety of indels observed. However, recent studies focusing on the common type 1 deletion and type 2 insertion mutations that account for approximately $80 \%$ of all indels have exhibited improved specificity and sensitivity necessary for MRD purposes [14].

2.1. JAK2 Mutations. As the JAK2 V617F is the most frequent mutation in MPN, considerable effort has been afforded in addressing the clinical usefulness of using this mutation as an
MRD marker in several treatment modalities. Hydroxyurea (HU) is one of the most widely used cytoreductive agents used in the treatment of PV and ET with initial reports suggesting that $\mathrm{HU}$ was able to suppress JAK2 V617F levels by $>30 \%$ in approximately half of patients, a phenomenon observed early in disease course $[15,16]$. Where partial molecular responses are recorded, they appear more likely in those patients with a high JAK2 V617F allele burden before treatment [17]. Subsequent studies with considerably longer follow-up, however, imply that HU does not appreciably reduce the JAK2 V617F allele burden which either remains stable or only exhibits minor fluctuations over prolonged treatment periods [18-20].

Interferon alpha (IFN- $\alpha$ ) is able to induce hematological responses in MPN patients but its use has been constrained by the side effects of the relatively large doses employed. Nonetheless, sustained molecular responses are observed in the majority of PV and ET patients who remain on treatment with complete elimination of the detectable JAK2 V617F clone in selected cases [21-23]. Rare cases have been described in which this complete molecular response is remarkably maintained for a number of years after cessation of IFN- $\alpha$ $[24,25]$. More recent clinical trial data point to less significant molecular responses in those patients with higher pretreatment allele burdens and in those with a higher incidence of disease modifying mutations [26]. Busulfan therapy for PV has lessened amid concerns of its leukemogenic potential; however, even in a small cohort of PV patients refractory to multiple therapies, busulfan induced considerable JAK2 V617F responses, including complete molecular remission, in the majority of patients [27].

The discovery that the JAK-STAT signalling pathway, required for normal hematopoietic signalling, is disrupted by mutations in the majority of MPN patients makes this pathway an obvious target for therapeutic intervention. Several inhibitors of both wild-type and mutant JAK1 and JAK2 molecules have been developed. Despite substantial improvements in reducing spleen size, improving constitutional symptoms, and prolonging survival in PMF patients, JAK inhibitors fail to significantly reduce the disease bulk as evidenced by a reduction of the JAK2 V617F load in those mutation positive patients $[28,29]$. However, with longer follow-up of these landmark clinical trials, $>10 \%$ of patients still on drug achieved either a partial or a complete molecular response with greater reductions in patients with shorter disease duration [30]. While the overall reduction in JAK2 V617F allele burden remains modest in most patients treated with JAK $1 / 2$ inhibitors, there is increasing evidence of slow but complete molecular responses in those patients on long term therapy justifying continued MRD assessment [31, 32].

Despite the introduction of JAK inhibitors for the treatment of PMF and HU-resistant PV, ASCT remains the only potentially curative option for those patients with advanced disease. Improvements in candidate patient selection and stratification, timing of transplantation, and conditioning regimens have significantly reduced the transplant related morbidity and increased the overall survival for patients undergoing this procedure [33]. Post-ASCT monitoring utilising additional patient-specific markers in addition to 
donor-recipient chimerism status is likely to provide a more beneficial, individualized approach. However, as relapse is a major cause of treatment failure after ASCT with salvage options limited and subsequent outcome relatively poor, identification of those patients at high-risk of relapse would be highly desirable, potentially enabling therapeutic intervention before overt relapse. Establishment of sensitive qPCR assays has retrospectively demonstrated the utility of this approach in detecting both disease clearance and persistence [10, 34-37]. Prospective monitoring allows consideration of preemptive adoptive immunotherapy with donor lymphocyte infusion in those cases with persistent or rising MRD [38, 39]. MRD assessment in the period immediately after ASCT should be considerably more frequent than in other modalities, perhaps on a weekly basis; one study has suggested that the level of MRD below or above $1.0 \%$ at one month after ASCT is highly predictive of outcome and relapse risk, respectively [40].

Mutations of JAK2 exon 12 are present in the small percentage of PV patients who are JAK2 V617F-negative. These JAK2 exon 12 mutations, which can be substitutions, deletions, insertions, and duplications, are usually detected with a low allelic burden of $<10 \%$ making quantitative MRD assessment problematic [41]. Individual qPCR assays for some common exon 12 mutations have been developed and demonstrate stability over time of both hetero-and homozygous allele burdens [42].

2.2. MPL Exon 10 Mutations. The most common MPL mutations are W515L and W515K with several other point mutations noted in codon W515 and elsewhere within exon 10 [43]. Although amenable to allele-specific qPCR approaches, their low frequency in ET and PMF, compared to the JAK2 V617F and CALR exon 9 mutations, has limited their use as MRD markers. In the post-ASCT setting, qPCR of both MPL W515L and W515A mutations in individual patients has demonstrated clearance of MRD in PMF patients [44, 45]. Lowering of $M P L \mathrm{~W} 515 \mathrm{~L} / \mathrm{K}$ allele burdens has also been demonstrated in ET patients treated with a telomerase inhibitor [46].

2.3. CALR Exon 9 Mutations. The diagnostic method of choice for CALR mutations in ET and PMF must have the ability to detect the plethora of indels thus far reported. Several approaches have been utilised with their sensitivities compared [47]; fragment length analysis (FLA) followed by capillary electrophoresis has been widely adopted and displays sensitivity compatible for MRD monitoring (approximately 1\%) but remains semiquantitative [48-50]. Analysis of CALR mutation by FLA has been retrospectively applied to patients with PMF after ASCT and has mirrored donor chimerism status in those relapsing patients demonstrating proof of principle [51]. In a cohort of $31 \mathrm{ET}$ patients with $C A L R$ mutations with considerable follow-up, interferon significantly decreased the CALR mutant allele burden with some patients achieving complete molecular remission; of further note, the presence of additional mutations was associated with a poorer molecular response [52]. In a retrospective analysis of a pivotal phase 3 study of PMF and post-ET PMF patients, a JAK $1 / 2$ inhibitor demonstrated clinical effectiveness in CALR-mutated patients but the allelic ratio as performed by FLA was not significantly lowered at a median of 60 weeks of treatment; however a small number of patients displayed molecular responses with longer follow-up [53].

In order to improve sensitivity and given the variety of CALR indels reported, qPCR approaches have focused on the two major mutations of type 1 ( $52 \mathrm{bp}$ deletion) and type 2 ( $5 \mathrm{bp}$ insertion) that account for more than $80 \%$ of $C A L R$ mutations. Initial attempts for qPCR of these common CALR mutations resulted in limited sensitivities of $1-2 \%$, comparable to other molecular screening approaches [54]. Development of alternative qPCR assays has managed to further improve the sensitivity for both type 1 and type 2 CALR mutations [14].

\section{Emerging Approaches}

3.1. Digital PCR. An emerging approach for the detection and quantitation of MPN-associated mutations is digital PCR (dPCR) which can achieve quantitation of the target allele without the requirement for standard curve construction or comparison to a reference gene. Partition of the template DNA into multiple PCR reactions is achieved by either droplet formation or nanofluidics, resulting in improved sensitivity and accuracy with minimal requirements for validation and standardization [55]. Comparisons of dPCR and qPCR have revealed strong correlations in the quantitation of the JAK2 V617F, even at low allele burdens of $0.1 \%$, indicating clinical utility for MRD monitoring [56-58]; however further studies are required to establish this capability.

The accurate determination of mutant CALR allele burdens by dPCR has recently been applied to PMF patients in the post-ASCT setting with considerable improvements observed in the detection and quantitation of type 1 and type 2 CALR mutants. As compared to FLA or qPCR, dPCR was capable of detecting delayed clearance of MRD after ASCT and earlier detection of increasing MRD levels prior to clinical relapse $[59,60]$. Sequential dPCR evaluations in a limited number MPN patients treated with interferon have shown reductions in the CALR mutant allele burden and, in one case, disappearance of the mutant clone at the time of transformation to acute myeloid leukemia [61].

3.2. Next-Generation Sequencing. The capacity of nextgeneration sequencing (NGS) technologies, platforms, and accompanying bioinformatics pipelines to simultaneously sequence multiple genes and identify mutations with high specificity and comparable sensitivity lends itself to MPN molecular diagnostics in which a considerable degree of genomic complexity occurs. Targeted exon sequencing allows the identification of several mutations that not only demonstrate clonality but also have prognostic and therapeutic significance $[62,63]$. While acquired mutations at diagnosis by NGS in myelodysplastic/myeloproliferative neoplasms may be used as subsequent markers of MRD after ASCT [64], the inadequate dynamic range of detection of NGS approaches 
has limited their assignment to routine MRD purposes. One encouraging study has demonstrated that increasing the number of NGS reads allows improved assessment of JAK2 V617F MRD levels and demonstrated appreciable correlation with a qPCR approach [65].

\section{Further Considerations}

Implementation of which MRD approach to adopt requires consideration of which cohort of patients to screen and technical aspects of the selected assays as discussed above. In addition harmonization of MRD data requires efficient handling and standardization of how results are to be reported [66] and also what options are available for both continued compliance and prospective proficiency evaluation. In order to audit this proficiency, internationally recognized standards are required. While an increased frequency of MRD assessment is required after ASCT the optimal testing frequency requires establishing for each therapeutic modality. Evaluation of standardized molecular responses is likely to be incorporated in clinical trials of novel targeted agents and potential drug combinations for the treatment of MPN, which is not reflected in current guidelines [67].

The identification of multiple gene defects and deregulated signalling pathways in MPN has, in turn, propagated expansion of clinical research into specific inhibitors targeting these aberrations. The addition of MRD monitoring is likely to supplement these interventions in providing an individualized pathway of patient management.

\section{Competing Interests}

The authors declare that there are no competing interests regarding the publication of this paper.

\section{References}

[1] R. C. Skoda, A. Duek, and J. Grisouard, "Pathogenesis of myeloproliferative neoplasms," Experimental Hematology, vol. 43, no. 8, pp. 599-608, 2015.

[2] S. E. Langabeer, H. Andrikovics, J. Asp et al., "Molecular diagnostics of myeloproliferative neoplasms," European Journal of Haematology, vol. 95, no. 4, pp. 270-279, 2015.

[3] H. L. Geyer and R. A. Mesa, "Emerging drugs for the treatment of myelofibrosis," Expert Opinion on Emerging Drugs, vol. 20, no. 4, pp. 663-678, 2015.

[4] A. J. Bench, H. E. White, L. Foroni et al., "Molecular diagnosis of the myeloproliferative neoplasms: UK guidelines for the detection of JAK2 V617F and other relevant mutations," British Journal of Haematology, vol. 160, no. 1, pp. 25-34, 2013.

[5] J. Z. Gong, J. R. Cook, T. C. Greiner et al., "Laboratory practice guidelines for detecting and reporting JAK2 and MPL mutations in myeloproliferative neoplasms: a report of the association for molecular pathology," Journal of Molecular Diagnostics, vol. 15, no. 6, pp. 733-744, 2013.

[6] E. Lippert, F. Girodon, E. Hammond et al., "Concordance of assays designed for the quantification of JAK2V617F: a multicenter study," Haematologica, vol. 94, no. 1, pp. 38-45, 2009.
[7] P. Collier, K. Patel, P. Waeltz et al., "Validation of standards for quantitative assessment of JAK2 c.1849G > T (p.V617F) allele burden analysis in clinical samples," Genetic Testing and Molecular Biomarkers, vol. 17, no. 5, pp. 429-437, 2013.

[8] E. Lippert, M. Boissinot, R. Kralovics et al., "The JAK2-V617F mutation is frequently present at diagnosis in patients with essential thrombocythemia and polycythemia vera," Blood, vol. 108, no. 6, pp. 1865-1867, 2006.

[9] T. S. Larsen, J. H. Christensen, H. C. Hasselbalch, and N. Pallisgaard, "The JAK2 V617F mutation involves Band T-lymphocyte lineages in a subgroup of patients with Philadelphia-chromosome negative chronic myeloproliferative disorders," British Journal of Haematology, vol. 136, no. 5, pp. 745-751, 2007.

[10] J. V. Jovanovic, A. Ivey, A. M. Vannucchi et al., "Establishing optimal quantitative-polymerase chain reaction assays for routine diagnosis and tracking of minimal residual disease in JAK2-V617F-associated myeloproliferative neoplasms: a joint European LeukemiaNet/MPN\&MPNr-EuroNet (COST action BM0902) study," Leukemia, vol. 27, no. 10, pp. 2032-2039, 2013.

[11] A. Pancrazzi, P. Guglielmelli, V. Ponziani et al., "A sensitive detection method for MPL W515L or MPL W515K mutation in chronic myeloproliferative disorders with locked nucleic acid-modified probes and real-time polymerase chain reaction," Journal of Molecular Diagnostics, vol. 10, no. 5, pp. 435-441, 2008.

[12] G.-R. Ruan, B. Jiang, L.-D. Li et al., "MPL W515L/K mutations in 343 Chinese adults with JAK2V617F mutation-negative chronic myeloproliferative disorders detected by a newly developed RQPCR based on TaqMan MGB probes," Hematological Oncology, vol. 28, no. 1, pp. 33-39, 2010.

[13] L. V. Furtado, H. C. Weigelin, K. S. J. Elenitoba-Johnson, and B. L. Betz, "Detection of MPL mutations by a novel allele-specific PCR-based strategy," Journal of Molecular Diagnostics, vol. 15, no. 6, pp. 810-818, 2013.

[14] M. Zinke, V. Nageswaran, R. Reinhardt, and T. Burmeister, "Rapid and sensitive detection of calreticulin type 1 and 2 mutations by real-time quantitative PCR," Molecular Diagnosis and Therapy, vol. 19, no. 5, pp. 329-334, 2015.

[15] F. Girodon, C. Schaeffer, C. Cleyrat et al., "Frequent reduction or absence of detection of the JAK2-mutated clone in JAK2V617Fpositive patients within the first years of hydroxyurea therapy," Haematologica, vol. 93, no. 11, pp. 1723-1727, 2008.

[16] A. Ricksten, L. Palmqvist, P. Johansson, and B. Andreasson, "Rapid decline of JAK2V617F levels during hydroxyurea treatment in patients with polycythemia vera and essential thrombocythemia," Haematologica, vol. 93, no. 8, pp. 1260-1261, 2008.

[17] C. Besses, A. Álvarez-Larrán, L. Martínez-Avilés et al., "Modulation of JAK2 V617F allele burden dynamics by hydroxycarbamide in polycythaemia vera and essential thrombocythaemia patients," British Journal of Haematology, vol. 152, no. 4, pp. 413419, 2011.

[18] A. Theocharides, J. R. Passweg, M. Medinger et al., "The allele burden of JAK2 mutations remains stable over several years in patients with myeloproliferative disorders," Haematologica, vol. 93, no. 12, pp. 1890-1893, 2008.

[19] T. S. Larsen, N. Pallisgaard, K. de Stricker, M. B. Møller, and H. C. Hasselbalch, "Limited efficacy of hydroxyurea in lowering of the JAK2 V617F allele burden," Hematology, vol. 14, no. 1, pp. 11-15, 2009. 
[20] E. Antonioli, A. Carobbio, L. Pieri et al., "Hydroxyurea does not appreciably reduce JAK2 v617F allele burden in patients with polycythemia vera or essential thrombocythemia," Haematologica, vol. 95, no. 8, pp. 1435-1438, 2010.

[21] J.-J. Kiladjian, B. Cassinat, S. Chevret et al., "Pegylated interferon-alfa-2a induces complete hematologic and molecular responses with low toxicity in polycythemia vera," Blood, vol. 112, no. 8, pp. 3065-3072, 2008.

[22] A. Quintás-Cardama, H. Kantarjian, T. Manshouri et al., "Pegylated interferon alfa-2a yields high rates of hematologic and molecular response in patients with advanced essential thrombocythemia and polycythemia vera," Journal of Clinical Oncology, vol. 27, no. 32, pp. 5418-5424, 2009.

[23] T. S. Larsen, K. F. Iversen, E. Hansen et al., "Long term molecular responses in a cohort of Danish patients with essential thrombocythemia, polycythemia vera and myelofibrosis treated with recombinant interferon alpha," Leukemia Research, vol. 37, no. 9, pp. 1041-1045, 2013.

[24] T. S. Larsen, O. W. Bjerrum, N. Pallisgaard, M. T. Andersen, M. B. Møller, and H. C. Hasselbalch, "Sustained major molecular response on interferon alpha-2b in two patients with polycythemia vera," Annals of Hematology, vol. 87, no. 10, pp. 847850, 2008.

[25] P. T. Murphy, S. McPherson, and S. E. Langabeer, "Complete molecular remission in a polycythaemia vera patient 12 years after discontinuation of interferon-alpha," Annals of Hematology, vol. 90, no. 2, pp. 233-234, 2011.

[26] A. Quintás-Cardama, O. Abdel-Wahab, T. Manshouri et al., "Molecular analysis of patients with polycythemia vera or essential thrombocythemia receiving pegylated interferon $\alpha$ 2a," Blood, vol. 122, no. 6, pp. 893-901, 2013.

[27] E. T. Kuriakose, S. Gjoni, Y. L. Wang et al., "JAK2 V617F allele burden is reduced by busulfan therapy: a new observation using an old drug," Haematologica, vol. 98, no. 11, pp. e135-e137, 2013.

[28] C. Harrison, J.-J. Kiladjian, H. K. Al-Ali et al., "JAK inhibition with ruxolitinib versus best available therapy for myelofibrosis," The New England Journal of Medicine, vol. 366, no. 9, pp. 787798, 2012.

[29] S. Verstovsek, R. A. Mesa, J. Gotlib et al., "A double-blind, placebo-controlled trial of ruxolitinib for myelofibrosis," The New England Journal of Medicine, vol. 366, no. 9, pp. 799-807, 2012.

[30] M. Deininger, J. Radich, T. C. Burn, R. Huber, D. Paranagama, and S. Verstovsek, "The effect of long-term ruxolitinib treatment on JAK2p.V617F allele burden in patients with myelofibrosis," Blood, vol. 126, no. 13, pp. 1551-1554, 2015.

[31] L. Pieri, A. Pancrazzi, A. Pacilli et al., "JAK2V617F complete molecular remission in polycythemia vera/essential thrombocythemia patients treated with ruxolitinib," Blood, vol. 125, no. 21, pp. 3352-3353, 2015.

[32] A. Koehler, K. Hubert, T. Lange et al., "JAK2V617F molecular remission in a primary myelofibrosis patient treated with ruxolitinib," Annals of Hematology, vol. 94, no. 11, pp. 1929-1930, 2015.

[33] N. M. Kröger, J. H. Deeg, E. Olavarria et al., "Indication and management of allogeneic stem cell transplantation in primary myelofibrosis: a consensus process by an EBMT/ELN international working group," Leukemia, vol. 29, no. 11, pp. 2126-2133, 2015.

[34] N. Kröger, A. Badbaran, E. Holler et al., "Monitoring of the JAK2-V617F mutation by highly sensitive quantitative real-time
PCR after allogeneic stem cell transplantation in patients with myelofibrosis," Blood, vol. 109, no. 3, pp. 1316-1321, 2007.

[35] N. K. Steckel, M. Koldehoff, M. Ditschkowski, D. W. Beelen, and A. H. Elmaagacli, "Use of the activating gene mutation of the tyrosine kinase (VAL617Phe) JAK2 as a minimal residual disease marker in patients with myelofibrosis and myeloid metaplasia after allogeneic stem cell transplantation," Transplantation, vol. 83, no. 11, pp. 1518-1520, 2007.

[36] H. Alchalby, A. Badbaran, T. Zabelina et al., "Impact of JAK2V617F mutation status, allele burden, and clearance after allogeneic stem cell transplantation for myelofibrosis," Blood, vol. 116, no. 18, pp. 3572-3581, 2010.

[37] K. Haslam, K. M. Molloy, E. Conneally, and S. E. Langabeer, "Evaluation of a JAK2 V617F quantitative PCR to monitor residual disease post-allogeneic hematopoietic stem cell transplantation for myeloproliferative neoplasms," Clinical Chemistry and Laboratory Medicine, vol. 52, no. 3, pp. e29-e31, 2014.

[38] O. Benjamini, M. Koren-Michowitz, N. Amariglio, N. Kroger, A. Nagler, and A. Shimoni, "Relapse of postpolycythemia myelofibrosis after allogeneic stem cell transplantation in a polycythemic phase: successful treatment with donor lymphocyte infusion directed by quantitative PCR test for V617F-JAK2 mutation," Leukemia, vol. 22, no. 10, pp. 1961-1963, 2008.

[39] N. Kröger, H. Alchalby, E. Klyuchnikov et al., "JAK2-V617F triggered preemptive and salvage adoptive immunotherapy with donor-lymphocyte infusion in patients with myelofibrosis after allogeneic stem cell transplantation," Blood, vol. 113, no. 8, pp. 1866-1868, 2009.

[40] T. Lange, A. Edelmann, U. Siebolts et al., "JAK2 p.V617F allele burden in myeloproliferative neoplasms one month after allogeneic stem cell transplantation significantly predicts outcome and risk of relapse," Haematologica, vol. 98, no. 5, pp. 722-728, 2013.

[41] S. Carillo, L. Henry, E. Lippert et al., "Nested high-resolution melting curve analysis: a highly sensitive, reliable, and simple method for detection of Jak2 exon 12 mutations-clinical relevance in the monitoring of polycythemia," Journal of Molecular Diagnostics, vol. 13, no. 3, pp. 263-270, 2011.

[42] L. Kjær, M. Westman, C. H. Riley, E. Høgdall, O. W. Bjerrum, and $\mathrm{H}$. Hasselbalch, "A highly sensitive quantitative real-time pcr assay for determination of mutant jak2 exon 12 allele burden," PLoS ONE, vol. 7, no. 3, Article ID e33100, 2012.

[43] W. Ma, X. Zhang, X. Wang et al., "MPL mutation profile in JAK2 mutation-negative patients with myeloproliferative disorders," Diagnostic Molecular Pathology, vol. 20, no. 1, pp. 34-39, 2011.

[44] E. Rumi, F. Passamonti, L. Arcaini et al., "Molecular remission after allo-SCT in a patient with post-essential thrombocythemia myelofibrosis carrying the MPL (W515A) mutation," Bone Marrow Transplantation, vol. 45, no. 4, pp. 798-800, 2010.

[45] H. Alchalby, A. Badbaran, O. Bock et al., "Screening and monitoring of MPL W515L mutation with real-time PCR in patients with myelofibrosis undergoing allogeneic-SCT," Bone Marrow Transplantation, vol. 45, no. 9, pp. 1404-1407, 2010.

[46] G. M. Baerlocher, E. O. Leibundgut, O. G. Ottmann et al., "Telomerase inhibitor imetelstat in patients with essential thrombocythemia," The New England Journal of Medicine, vol. 373, no. 10, pp. 920-928, 2015.

[47] A. V. Jones, D. Ward, M. Lyon et al., "Evaluation of methods to detect CALR mutations in myeloproliferative neoplasms," Leukemia Research, vol. 39, no. 1, pp. 82-87, 2015.

[48] T. Klampfl, H. Gisslinger, A. S. Harutyunyan et al., "Somatic mutations of calreticulin in myeloproliferative neoplasms," The 
New England Journal of Medicine, vol. 369, no. 25, pp. 23792390, 2013.

[49] Q.-M. Yao, J. Zhou, R. P. Gale et al., "A rapid, highly accurate method for quantifying calr mutant allele burden in persons with myeloproliferative neoplasms," Hematology, vol. 20, no. 9, pp. 517-522, 2015.

[50] M. Mehrotra, R. Luthra, R. R. Singh et al., "Clinical validation of a multipurpose assay for detection and genotyping of CALR mutations in myeloproliferative neoplasms," American Journal of Clinical Pathology, vol. 144, no. 5, pp. 746-755, 2015.

[51] K. Haslam, S. E. Langabeer, K. Molloy, M. F. Mcmullin, and E. Conneally, "Assessment of CALR mutations in myelofibrosis patients, post-allogeneic stem cell transplantation," British Journal of Haematology, vol. 166, no. 5, pp. 800-802, 2014.

[52] E. Verger, B. Cassinat, A. Chauveau et al., "Clinical and molecular response to interferon- $\alpha$ therapy in essential thrombocythemia patients with CALR mutations," Blood, vol. 126, no. 24, pp. 2585-2591, 2015.

[53] P. Guglielmelli, G. Rotunno, C. Bogani et al., "Ruxolitinib is an effective treatment for CALR-positive patients with myelofibrosis," British Journal of Haematology, vol. 173, no. 6, pp. 938-940, 2016.

[54] J. Chi, M. Manoloukos, C. Pierides et al., "Calreticulin mutations in myeloproliferative neoplasms and new methodology for their detection and monitoring," Annals of Hematology, vol. 94, no. 3, pp. 399-408, 2014.

[55] J. F. Huggett, S. Cowen, and C. A. Foy, "Considerations for digital PCR as an accurate molecular diagnostic tool," Clinical Chemistry, vol. 61, no. 1, pp. 79-88, 2015.

[56] E. Kinz, A. Leiherer, A. H. Lang, H. Drexel, and A. Muendlein, "Accurate quantitation of JAK2 V617F allele burden by arraybased digital PCR," International Journal of Laboratory Hematology, vol. 37, no. 2, pp. 217-224, 2015.

[57] G. Fontanelli, C. Baratè, E. Ciabatti et al., "Real-Time PCR and Droplet Digital PCR: two techniques for detection of the JAK2 (V617F) mutation in Philadelphia-negative chronic myeloproliferative neoplasms," International Journal of Laboratory Hematology, vol. 37, no. 6, pp. 766-773, 2015.

[58] M. Waterhouse, M. Follo, D. Pfeifer et al., "Sensitive and accurate quantification of JAK2 V617F mutation in chronic myeloproliferative neoplasms by droplet digital PCR," Annals of Hematology, vol. 95, no. 5, pp. 739-744, 2016.

[59] O. Mansier, M. Migeon, A. Saint-Lézer et al., "Quantification of the mutant CALR allelic burden by digital PCR: application to minimal residual disease evaluation after bone marrow transplantation," Journal of Molecular Diagnostics, vol. 18, no. 1, pp. 68-74, 2016.

[60] A. Badbaran, B. Fehse, M. Christopeit et al., "Digital-PCR assay for screening and quantitative monitoring of calreticulin (CALR) type-2 positive patients with myelofibrosis following allogeneic stem cell transplantation," Bone Marrow Transplantation, vol. 51, no. 6, pp. 872-873, 2016.

[61] L. Anelli, A. Zagaria, N. Coccaro et al., "Droplet digital PCR assay for quantifying of CALR mutant allelic burden in myeloproliferative neoplasms," Annals of Hematology, vol. 95, no. 9, pp. 1559-1560, 2016.

[62] P. Lundberg, A. Karow, R. Nienhold et al., "Clonal evolution and clinical correlates of somatic mutations in myeloproliferative neoplasms," Blood, vol. 123, no. 14, pp. 2220-2228, 2014.

[63] M. M. J. Kirschner, M. Schemionek, C. Schubert et al., "Dissecting genomic aberrations in myeloproliferative neoplasms by multiplex-PCR and next generation sequencing," PLoS ONE, vol. 10, no. 4, article e0123476, 2015.

[64] Y. Fu, T. Schroeder, T. Zabelina et al., "Postallogeneic monitoring with molecular markers detected by pretransplant nextgeneration or Sanger sequencing predicts clinical relapse in patients with myelodysplastic/myeloproliferative neoplasms," European Journal of Haematology, vol. 92, no. 3, pp. 189-194, 2014.

[65] E. Abdelhamid, M. Figeac, A. Renneville et al., "Quantification of JAK2V617F mutation by next-generation sequencing technology," American Journal of Hematology, vol. 88, no. 6, pp. 536537, 2013.

[66] M. Ostergaard, C. G. Nyvold, J. V. Jovanovic et al., "Development of standardized approaches to reporting of minimal residual disease data using a reporting software package designed within the European LeukemiaNet," Leukemia, vol. 25, no. 7, pp. 1168-1173, 2011.

[67] G. Barosi, A. Tefferi, C. Besses et al., "Clinical end points for drug treatment trials in BCR-ABL1-negative classic myeloproliferative neoplasms: consensus statements from European LeukemiaNET (ELN) and Internation Working GroupMyeloproliferative Neoplasms Research and Treatment (IWGMRT)," Leukemia, vol. 29, no. 1, pp. 20-26, 2015. 


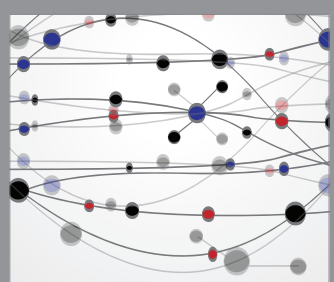

The Scientific World Journal
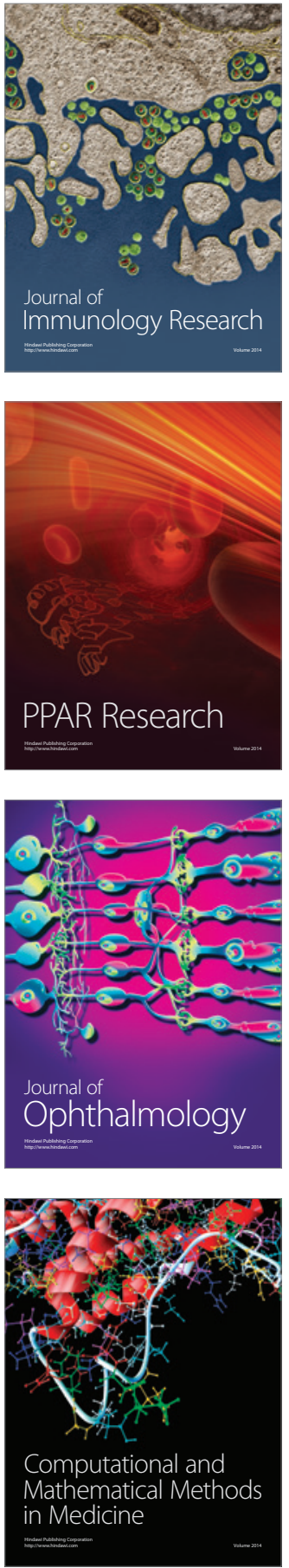

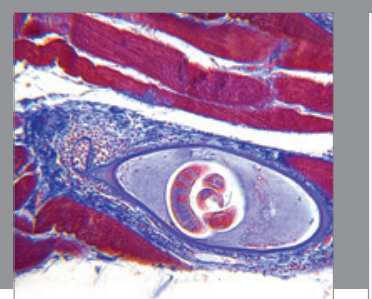

Gastroenterology Research and Practice

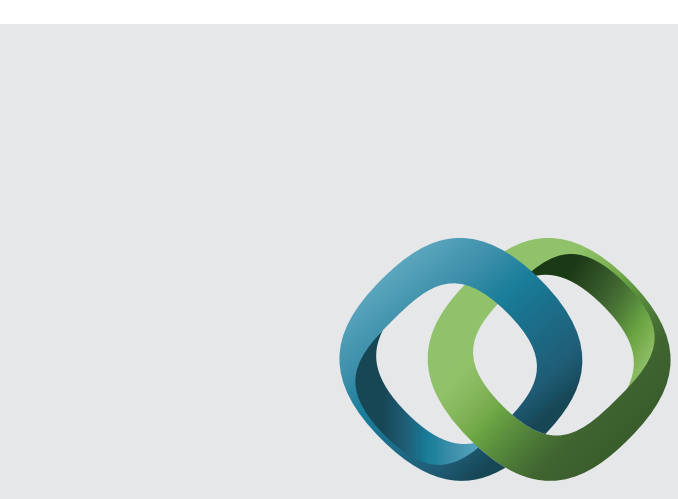

\section{Hindawi}

Submit your manuscripts at

http://www.hindawi.com
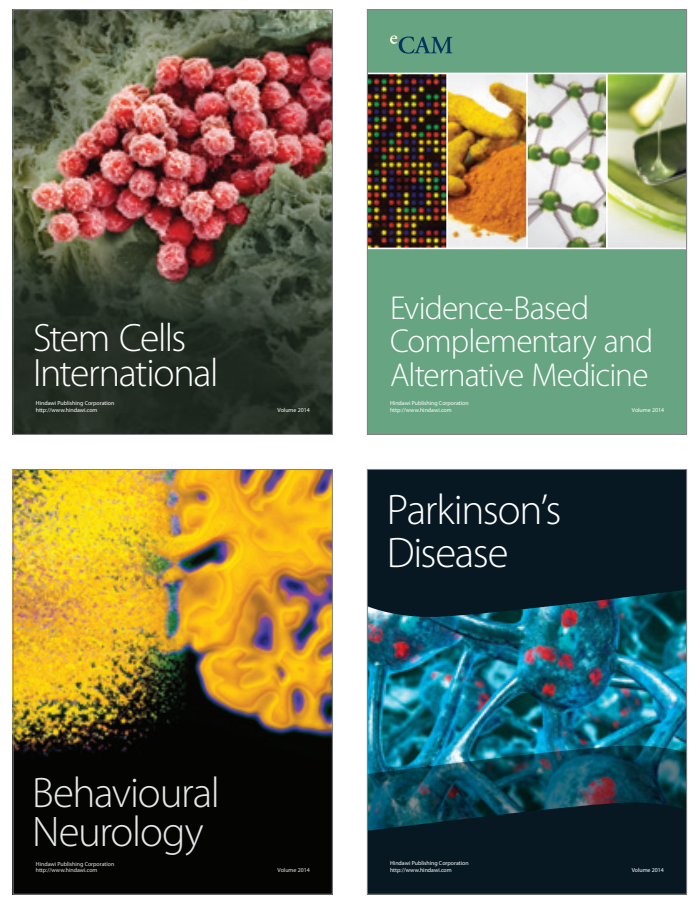
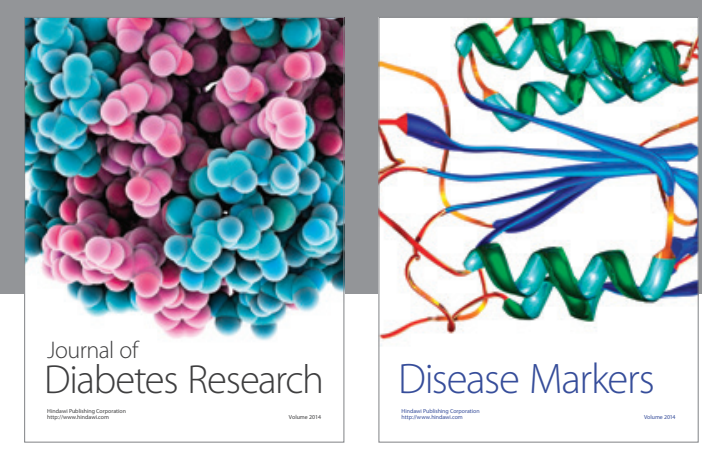

Disease Markers
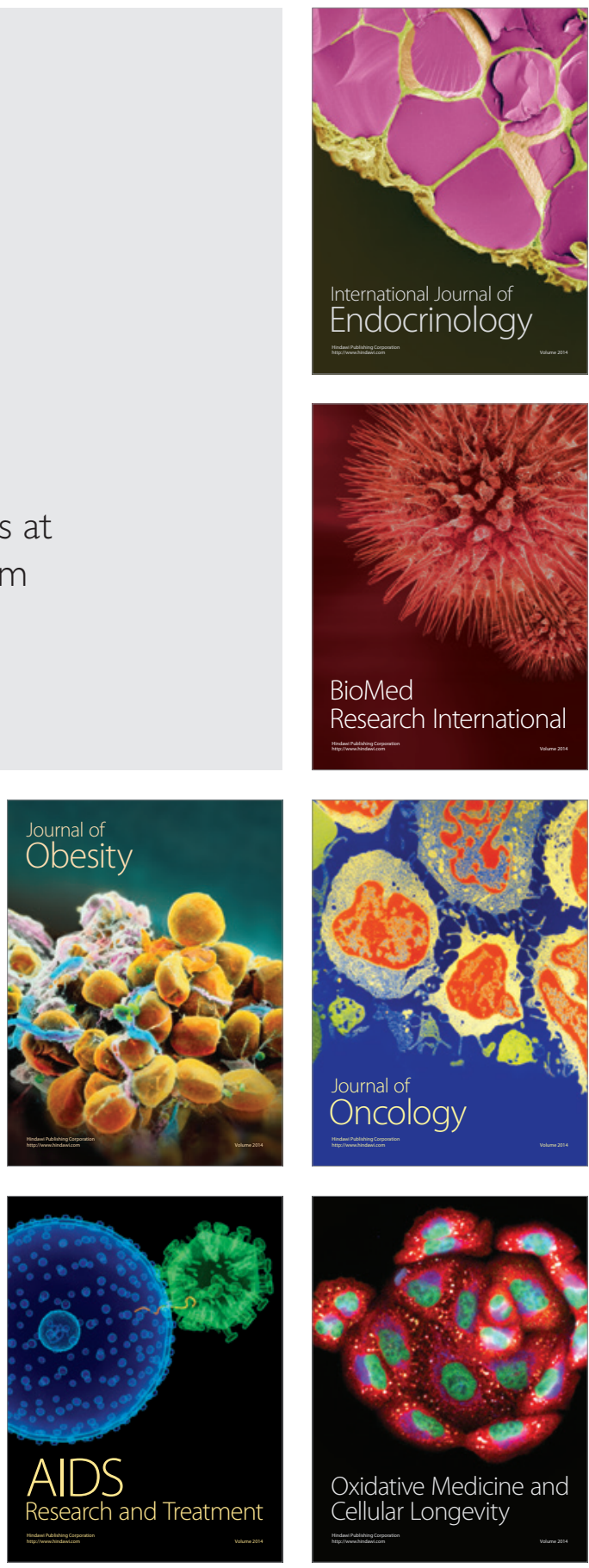